\title{
Non-Invasive Brain-Actuated Interaction
}

\author{
José del R. Millán ${ }^{1,2}$, Pierre W. Ferrez ${ }^{1,2}$, Ferran Galán ${ }^{1,2}$, Eileen Lew ${ }^{1,2}$, \\ Ricardo Chavarriaga ${ }^{1,2}$ \\ ${ }^{1}$ IDIAP Research Institute, Rue du Simplon 4, 1920 Martigny, Switzerland \\ ${ }^{2}$ Ecole Polytechnique Fédérale de Lausanne (EPFL), Switzerland \\ \{jose.millan, pierre.ferrez, ferran.galan, eileen.lew, ricardo.chavarriaga\}@idiap.ch
}

\begin{abstract}
The promise of Brain-Computer Interfaces (BCI) technology is to augment human capabilities by enabling interaction with computers through a conscious and spontaneous modulation of the brainwaves after a short training period. Indeed, by analyzing brain electrical activity online, several groups have designed brain-actuated devices that provide alternative channels for communication, entertainment and control. Thus, a person can write messages using a virtual keyboard on a computer screen and also browse the internet. Alternatively, subjects can operate simple computer games, or brain games, and interact with educational software. Work with humans has shown that it is possible for them to move a cursor and even to drive a wheelchair. This paper briefly reviews the field of BCI, with a focus on non-invasive systems based on electroencephalogram (EEG) signals. It also describes three brain-actuated devices we have developed: a virtual keyboard, a brain game, and a wheelchair. Finally, it shortly discusses current research directions we are pursuing in order to improve the performance and robustness of our BCI system, especially for real-time control of brain-actuated robots.
\end{abstract}

Keywords: Brain-computer interfaces, electroencephalogram, asynchronous protocols, brain-actuated devices, statistical classifiers, feature selection.

\section{Introduction}

The idea of controlling machines not by manual operation, but by mere "thinking" (i.e., the brain activity of human subjects) has fascinated humankind since ever, and researchers working at the crossroads of computer science, neurosciences, and biomedical engineering have started to develop the first prototypes of brain-computer interfaces (BCI) over the last decade or so [1], [2], [3], [4], [5]. A BCI monitors the user's brain activity and translates their intentions into actions - such as moving a wheelchair [6], [7] or selecting a letter from a virtual keyboard [8], [9] — without using activity of any muscle or peripheral nerve. The central tenet of a BCI is the capability to distinguish different patterns of brain activity, each being associated to a particular intention or mental task.

Such a kind of BCI is a natural way to augment human capabilities by providing a new interaction link with the outside world and is particularly relevant as an aid for paralyzed humans, although it also opens up new possibilities in natural and direct 
interaction for able-bodied people. Figure 1 shows the general architecture of a BCI. Brain electrical activity is recorded with a portable device. These raw signals are first processed and transformed in order to extract some relevant features that are then passed on to some mathematical models (e.g., statistical classifiers or neural networks). This model computes, after some training process, the appropriate mental commands to control the device. Finally, visual feedback, and maybe other kinds such as tactile stimulation, informs the subject about the performance of the brain-actuated device so that they can learn appropriate mental control strategies and make rapid changes to achieve the task.

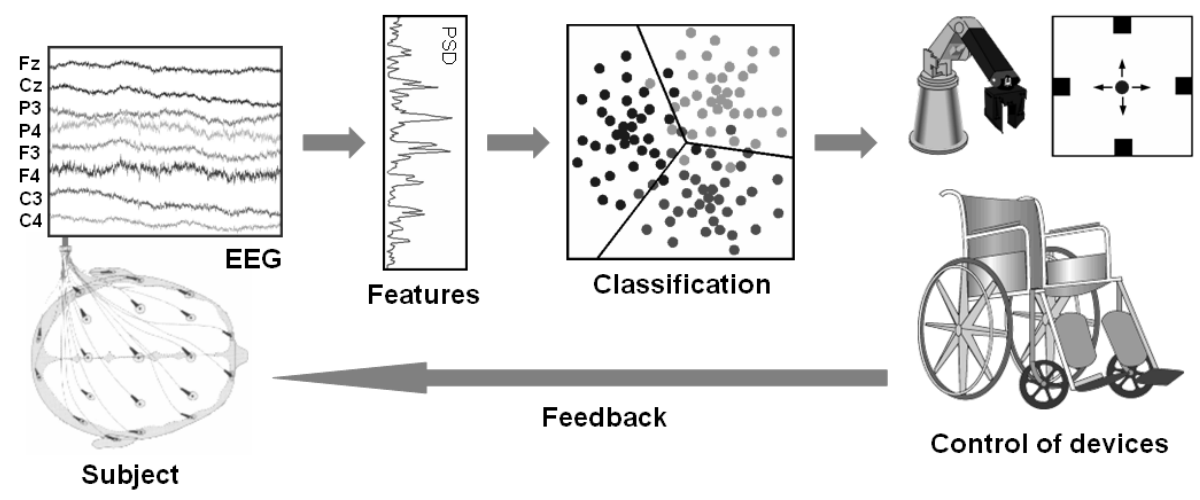

Fig. 1. General architecture of a brain-computer interface (BCI) for controlling devices such as a cursor, a robotic arm, or a motorized wheelchair. In this case the BCI measures electroencephalogram (EEG) signals recorded non-invasively from electrodes placed on the subject's scalp.

A BCI may monitor brain activity via a variety of methods, which can be coarsely classified as invasive and non-invasive. In invasive BCI systems the activity of single neurons (their spiking rate) is recorded from microelectrodes implanted in the brain. Less invasive approaches are based on the analysis of electrocorticogram (ECoG) signals from electrodes implanted under the skull. For humans, however, it is preferable to use non-invasive approaches to avoid the risks generated by permanent surgically implanted devices in the brain, and the associated ethical concerns. Most non-invasive BCI systems use electroencephalogram (EEG) signals; i.e., the electrical brain activity recorded from electrodes placed on the scalp. The main source of the EEG is the synchronous activity of thousands of cortical neurons. Measuring the EEG is a simple noninvasive way to monitor electrical brain activity, but it does not provide detailed information on the activity of single neurons (or small brain areas). Moreover, it is characterized by small signal amplitudes (a few $\mu$ Volts) and noisy measurements (especially if recording outside shield rooms).

Besides electrical activity, neural activity also produces other types of signals, such as magnetic and metabolic, that could be used in a BCI. Magnetic fields can be recorded with magnetoencephalography (MEG), while brain metabolic activityreflected in changes in blood flow-can be observed with positron emission 
tomography (PET), functional magnetic resonance imaging (fMRI), and optical imaging. Unfortunately, such alternative techniques require sophisticated devices that can be operated only in special facilities. Moreover, techniques for measuring blood flow have long latencies and thus are less appropriate for interaction.

From this short review it follows that, because of its low cost, portability and lack of risk, EEG is the ideal modality if we want to bring BCI technology to a large population.

In the next sections we review the main components of our BCI system, which is based on the online analysis of spontaneous EEG signals and recognizes 3 mental tasks. Our approach relies on three principles. The first one is an asynchronous protocol where subjects decide voluntarily when to switch between mental tasks and perform those mental tasks at their own pace. The second principle is mutual learning, where the user and the BCI are coupled together and adapt to each other. In other words, we use machine learning approaches to discover the individual EEG patterns characterizing the mental tasks executed by the user while users learn to modulate their brainwaves so as to improve the recognition of the EEG patterns. Finally, the third principle is the combination of the user's intelligence with the design of intelligent devices that facilitate interaction and reduce the user's cognitive workload. This is particularly useful for mental control of robots. We also describe the three brain-actuated applications we have developed. Finally, we discuss current research directions we are pursuing in order to improve the performance and robustness of our BCI system, especially for real-time control of brain-actuated robots.

\section{Spontaneous EEG and Asynchronous Operation}

Non-invasive EEG-based BCIs can be classified as "evoked" or "spontaneous". An evoked BCI exploits a strong characteristic of the EEG, the so-called evoked potential, which reflects the immediate automatic responses of the brain to some external stimuli. Evoked potentials are, in principle, easy to pick up with scalp electrodes. The necessity of external stimulation does, however, restrict the applicability of evoked potentials to a limited range of tasks. In our view, a more natural and suitable alternative for interaction is to analyze components associated with spontaneous "intentional" mental activity. This is particularly the case when controlling robotics devices. Spontaneous BCIs are based on the analysis of EEG phenomena associated with various aspects of brain function related to mental tasks carried out by the subject at his/her own will. Such a kind of BCI can exploit two kinds of spontaneous, or endogenous, brain signals, namely slow potential shifts [10] or variations of rhythmic activity [6], [8], [11], [12], [13], [14]. We will focus on the latter that are the most common.

EEG-based BCIs are limited by a low channel capacity ${ }^{1}$. Most of the current systems have a channel capacity below $0.5 \mathrm{bits} / \mathrm{s}$ [3]. One of the main reasons for such a low bandwidth is that they are based on synchronous protocols where EEG is time-locked to externally paced cues repeated every 4-10 s and the response of the

${ }^{1}$ Channel capacity is the maximum possible information transfer rate, or bit rate, through a channel. 
BCI is the overall decision over this period [10], [12], [13]. Such synchronous protocols facilitate EEG analysis since the starting time of mental states are precisely known and differences with respect to background EEG activity can be amplified. Unfortunately, they are slow and BCI systems that use them normally recognize only 2 mental states.

On the contrary, we utilize more flexible asynchronous protocols where the subject makes self-paced decisions on when to stop doing a mental task and start immediately the next one [6], [8], [15]. In such asynchronous protocols the subject can voluntarily change the mental task being executed at any moment without waiting for external cues. The time of response of an asynchronous BCI can be below 1 second. For instance, in our approach the system responds every $1 / 2$ second. The rapid responses of our asynchronous BCI, together with its performance (see Section 3), give a theoretical channel capacity between 1 and $1.5 \mathrm{bits} / \mathrm{s}$.

\section{The Machine Learning Way to BCI}

A critical issue for the development of a $\mathrm{BCI}$ is training-i.e., how users learn to operate the BCI. Some groups have demonstrated that some subjects can learn to control their brain activity through appropriate, but lengthy, training in order to generate fixed EEG patterns that the BCI transforms into external actions [10], [13]. In this case the subject is trained over several months to modify the amplitude of their EEG signals. We follow a mutual learning process to facilitate and accelerate the user's training period. Indeed, our approach allows subjects to achieve good performances in just a few hours of training in the presence of feedback [8].

Most BCI systems deal with the recognition of just 2 mental tasks [11], [12], [14], [15]. Our approach achieves error rates below $5 \%$ for 3 mental tasks, but correct recognition is $70 \%$. In the remaining cases (around 20-25\%), the classifier doesn't respond, since it considers the EEG samples as uncertain. The incorporation of rejection criteria (see below) to avoid making risky decisions is an important concern in BCI. From a practical point of view, a low classification error is a critical performance criterion for a $\mathrm{BCI}$; otherwise users can become frustrated and stop utilizing it.

We use machine learning techniques at two levels, namely feature selection and training the classifier embedded into the BCI. The approach aims at discovering subject-specific spatio-frequency patterns embedded in the continuous EEG signali.e., EEG rhythms over local cortical areas that differentiate the mental tasks. At the first level, we select those features that are more relevant for discriminating among the mental tasks. The selected features are those that satisfy two criteria: maximization of the separability of the mental tasks and stability over time. Indeed, EEG signals are non-stationary and, so, change over time. Feature selection is based on canonical variates analysis [16]. This procedure yields a sample, or input vector, $\mathbf{x}$ composed of the power of some frequency components from some electrodes.

We use a statistical Gaussian classifier (see [6] for more details). The output of this statistical classifier is an estimation of the posterior class probability distribution for a sample; i.e., the probability that a given single trial belongs to each mental task (or 
class). Each class is represented by a number of Gaussian prototypes, typically less than four. That is, we assume that the class-conditional probability function of class $C_{k}$ is a superposition of $N_{k}$ Gaussian prototypes. We also assume that all classes have equal prior probability. All classes have the same number of prototypes $N_{p}$, and for each class each prototype has equal weight $1 / N_{k}$. Then, dropping constant terms, the activity $a_{k}^{i}$ of the $i^{\text {th }}$ prototype of class $C_{k}$ for a given sample $\mathbf{x}$ is the value of the

Gaussian with centre $\mu_{k}^{i}$ and covariance matrix $\Sigma_{k}^{i}$. From this we calculate the posterior probability $y_{k}$ of the class $C_{k}$. The posterior probability $y_{k}$ of the class $C_{k}$ is now the sum of the activities of all the prototypes of class $k$ divided by the sum of the activities of all the prototypes of all the classes.

The classifier output for input vector $\mathbf{x}$ is now the class with the highest probability, provided that the probability is above a given threshold, otherwise the result is "unknown".

Usually each prototype of each class would have an individual covariance matrix $\Sigma_{k}^{i}$, but to reduce the number of parameters the model has a single diagonal covariance matrix common to all the prototypes of the same class. During offline training of the classifier, the prototype centers are initialized by any clustering algorithm or generative approach. This initial estimate is then improved by stochastic gradient descent to minimize the mean square error $E=1 / 2 \sum_{k}\left(y_{k}-t_{k}\right)^{2}$, where $\mathbf{t}$ is the target vector in the form 1-of-C; that is, if the second of three classes was the desired output, the target vector is $(0,1,0)$. The covariance matrices are computed individually and are then averaged over the prototypes of each class to give $\Sigma_{k}$.

\section{Hardware and Signal Acquisition}

We acquire EEG potentials with a portable BioSemi system using a cap with either 32 or 64 integrated electrodes arranged in the modified 10/20 International System. The EEG recordings are monopolar and taken at $512 \mathrm{~Hz}$.

EEG signals are characterized by a poor signal-to-noise ratio and spatial resolution. Their quality is greatly improved by means of spatial filtering techniques. We use the common average reference (CAR) procedure, where at each time step the average potential over all the channels is subtracted from each channel. This re-referencing procedure removes the background activity, leaving activity from local sources beneath the electrodes. Alternatively, raw EEG potentials can be transformed by means of a Surface Laplacian (SL) derivation. The SL estimate yields new potentials that represent better the cortical activity originated in radial sources immediately below the electrodes. The superiority of SL- and/or CAR-transformed signals over raw potentials for the operation of a BCI has been demonstrated in different studies [11], [17]. 


\section{Brain-Actuated Devices}

BCI systems are being used to operate a number of brain-actuated applications that augment people's communication capabilities, provide new forms of entertainment, and also enable the operation of physical devices. In this section we briefly describe some of the brain-actuated devices we have developed over the years. All these systems have been largely demonstrated publicly.

Our asynchronous BCI can be used to select letters from a virtual keyboard on a computer screen and to write a message [8], [9]. Initially, the whole keyboard (26 English letters plus the space to separate words, for a total of 27 symbols organized in a matrix of 3 rows by 9 columns) is divided in three blocks, each associated to one of the mental tasks. The association between blocks and mental tasks is indicated by the same colors as during the training phase. Each block contains an equal number of symbols, namely 9 at this first level ( 3 rows by 3 columns). Then, once the statistical classifier recognizes the block on which the subject is concentrating, this block is split in 3 smaller blocks, each having 3 symbols this time ( 1 row). As one of this secondlevel blocks is selected, it is again split in 3 parts. At this third and final level, each block contains 1 single symbol. Finally, to select the desired symbol, the user concentrates in its associated mental task as indicated by the color of the symbol. This symbol goes to the message and the whole process starts over again. Thus, the process of writing a single letter requires three decision steps.

The second brain-actuated device is a simple computer game [9], or "brain game", but other educational software could have been selected instead. It is the classical Pacman. For the control of Pacman, two mental tasks are enough to make it turn left of right. Pacman changes direction of movement whenever one of the mental tasks is recognized twice in a row. In the absence of further mental commands, Pacman moves forward until it reaches a wall, where it stops and waits for instructions.

Finally, it is also possible to control mentally robots and prosthesis. Until recently, EEG-based BCIs have been considered too slow for controlling rapid and complex sequences of movements. But we have shown for the first time [6], [8] that asynchronous analysis of EEG signals is sufficient for humans to continuously control a mobile robot - emulating a motorized wheelchair-along non-trivial trajectories requiring fast and frequent switches between mental tasks (see Fig. 2). Two human subjects learned to mentally drive the robot between rooms in a house-like environment visiting 3 or 4 rooms in the desired order. Furthermore, mental control was only marginally worse than manual control on the same task. A key element of this brain-actuated robot is shared control between two intelligent agents - the human user and the robot - so that the user only gives high-level mental commands that the robot performs autonomously. In particular, the user's mental states are associated with high-level commands (e.g., "turn right at the next occasion") and that the robot executes these commands autonomously using the readings of its on-board sensors. Another critical feature is that a subject can issue high-level commands at any moment. This is possible because the operation of the BCI is asynchronous and, unlike synchronous approaches, does not require waiting for external cues. The robot relies on a behaviour-based controller to implement the high-level commands to guarantee obstacle avoidance and smooth turns. In this kind of controller, on-board sensors are read constantly and determine the next action to take. 




Fig. 2. One of the users while driving mentally the robot through the different rooms of the environment, making it turn right, turn left, or move forward. The robot has 3 lights on top to provide feedback to the user and 8 infrared sensors around its diameter to detect obstacles.

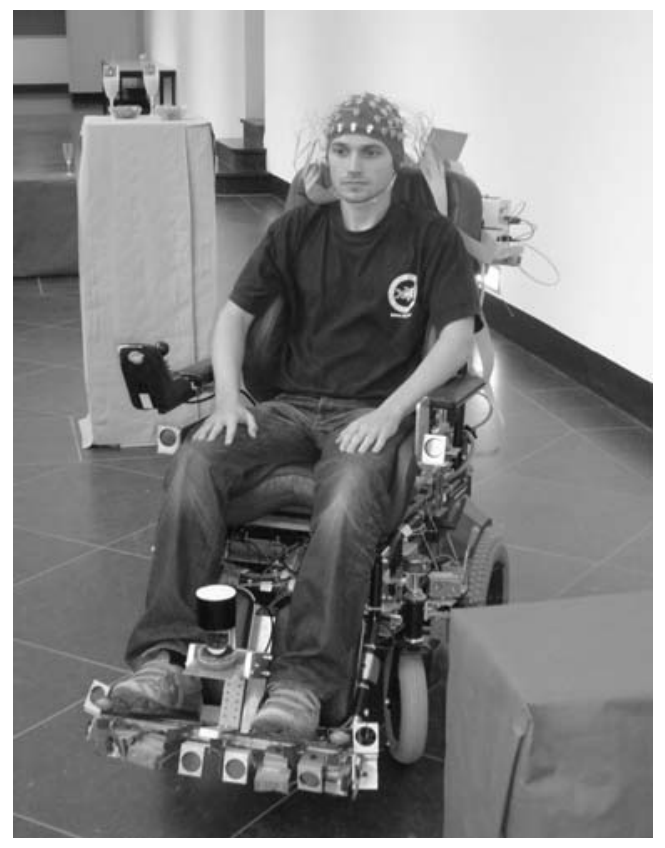

Fig. 3. Subject driving the wheelchair in a natural environment from non-invasive EEG. Note the laser scanner in front of the wheelchair, in between the subject's legs. 
More recently, we have extended this work to the mental control of both a simulated and a real wheelchair (see Fig. 3). This has been done in the framework of the European project MAIA (http://www.maia-project.org) and in cooperation with the KU Leuven. In this case, we have incorporated shared control principles into the BCI [18], [19]. In shared control, the intelligent controller relieves the human from low level tasks without sacrificing the cognitive superiority and adaptability of human beings that are capable of acting in unforeseen situations. In other words, in shared control there are two intelligent agents - the human user and the robot-so that the user only conveys intents that the robot performs autonomously. Although our first brain-actuated robot had already some form of cooperative control, shared autonomy is a more principled and flexible framework. Shared autonomy is also an essential component of any high-performance brain-actuated space device of the future.

\section{Current Directions of Research}

For brain-actuated robots, contrarily to augmented communication through BCI, fast decision-making is critical. In this sense, real-time control of brain-actuated devices, especially robots and neuroprostheses, is the most challenging application for BCI. While brain-actuated robots have been demonstrated in the laboratory, this technology is not yet ready to be taken out and used in real-world situations. A critical issue is how to improve the robustness of BCIs with the goal of making it a more practical and reliable technology. A first avenue of research is online adaptation of the interface to the user to keep the BCI constantly tuned to its owner [20], [21]. The point here is that, as subjects gain experience, they develop new capabilities and change their brain activity patterns. In addition, brain signals change naturally over time. In particular, this is the case from a session (with which data the classifier is trained) to the next (where the classifier is applied). Thus, online learning can be used to adapt the classifier throughout its use and keep it tuned to drifts in the signals it is receiving in each session. Preliminary work shows the feasibility and benefits of this approach.

The second line is the analysis of neural correlates of high-level cognitive and affective states such as errors, alarms, attention, frustration, confusion, etc. Information about these states is embedded in the EEG together with the mental commands intentionally generated by the user. The ability to detect and adapt to these states would enable the BCI to interact with the user in a much more meaningful way. One of these high-level states is the awareness of erroneous responses, whose neural correlate arises in the millisecond range. Thus, user's commands are executed only if no error is detected in this short time. Recent results have shown satisfactory singletrial recognition of errors that leads to significant improvement of the BCI performance [22], [23]. In addition, this new type of error potential-which is generated in response to errors made by the BCI rather than by the user-can provide with performance feedback that, in combination with online adaptation, allows improving the BCI while it is being used [24]. 
Acknowledgments. This work is supported by the Swiss National Science Foundation through the National Centre of Competence in Research on "Interactive Multimodal Information Management (IM2)" and also by the European IST Programme FET Project FP6-003758. This paper only reflects the authors' views and funding agencies are not liable for any use that may be made of the information contained herein.

\section{References}

1. Nicolelis, M.A.L.: Actions from Thoughts. Nature 409, 403-407 (2001)

2. Millán, J.d.R.: Brain-Computer Interfaces. In: Arbib, M.A. (ed.) Handbook of Brain Theory and Neural Networks, pp.178-181. MIT Press, Cambridge, Massachusetts (2002)

3. Wolpaw, J.R., Birbaumer, N., McFarland, D.J., Pfurtscheller, G., Vaughan, T.M.: BrainComputer Interfaces for Communication and Control. Clin. Neurophysiol. 113, 767-791 (2002)

4. Wickelgren, I:. Tapping the Mind. Science 299, 496-499 (2003)

5. Dornhege, G., Millán, J.d.R., Hinterberger, T., McFarland, D., Müller, K.-R. (eds.): Towards Brain-Computer Interfacing. MIT Press, Cambridge, Massachusetts (2007)

6. Millán, J.d.R., Renkens, F., Mouriño, J., Gerstner, W.: Non-Invasive Brain-Actuated Control of a Mobile Robot by Human EEG. IEEE Trans. Biomed. Eng. 51, 1026-1033 (2004)

7. Galán, F., Nuttin, M., Lew, E., Ferrez, P.W., Vanacker, G., Philips, J., Van Brussel, H., Millán, J.d.R.: An Asynchronous and Non-Invasive Brain-Actuated Wheelchair. In: 13th International Symposium on Robotics Research. Hirsoshima, Japan (2007)

8. Millán, J.d.R., Renkens, F., Mouriño, J., Gerstner, W.: Brain-Actuated Interaction. Artif. Intell. 159, 241-259 (2004)

9. Millán, J.d.R.: Adaptive Brain Interfaces. Comm. ACM 46, 74-80 (2003)

10. Birbaumer, N., Ghanayim, N., Hinterberger, T., Iversen, I., Kotchoubey, B., Kübler, A., Perelmouter, J., Taub, E., Flor, H.: A Spelling Device for the Paralysed. Nature 398, $297-$ 298 (1999)

11. Babiloni, F., Cincotti, F., Lazzarini, L., Millán, J.d.R., Mouriño, J., Varsta, M., Heikkonen, J., Bianchi, L., Marciani, M.G.: Linear Classification of Low-Resolution EEG Patterns Produced by Imagined Hand Movements. IEEE Trans. Rehab. Eng. 8, 186-188 (2000)

12. Pfurtscheller, G., Neuper, C.: Motor Imagery and Direct Brain-Computer Communication. Proc. IEEE 89, 1123-1134 (2001)

13. Wolpaw, J.R., McFarland, D.J.: Control of a Two-Dimensional Movement Signal by a Noninvasive Brain-Computer Interface in Humans. PNAS 101, 17849-17854 (2004)

14. Blankertz, B., Dornhege, G., Krauledat, M, Müller, K.R., Kunzmann, V., Losch, F., Curio, G.: The Berlin Brain-Computer Interface: EEG-based Communication without Subject Training. IEEE Trans. Neural Sys. Rehab. Eng. 14, 147-152 (2006)

15. Birch, G.E., Bozorgzadeh, Z., Mason, S.G.: Initial On-Line Evaluation of the LF-ASD Brain-Computer Interface with Able-Bodied and Spinal-Cord Subjects using Imagined Voluntary Motor Potentials. IEEE Trans. Neural Sys. Rehab. Eng. 10, 219-224 (2002)

16. Galán, F., Ferrez, P.W., Oliva, F, Guàrdia, J., Millán, J.d.R.: Feature Extraction for Multiclass BCI using Canonical Variates Analysis. In: IEEE International Symposium on Intelligent Signal Processing. Alcalá de Henares, Spain (2007)

17. Mouriño, J.: EEG-based Analysis for the Design of Adaptive Brain Interfaces. Ph.D. thesis, Centre de Recerca en Enginyeria Biomèdica, Universitat Politècnica de Catalunya, Barcelona, Spain (2003) 
18. Philips, J., .Millán, J.d.R., Vanacker, G, Lew, E., Galán, F., Ferrez, P.W., Van Brussel, H., Nuttin, M.: Adaptive Shared Control of a Brain-Actuated Simulated Wheelchair. In: 10th International Conference on Rehabilitation Robotics, Noordwijk, The Netherlands (2007)

19. Vanacker, G, Millán, J.d.R., Lew, E., Ferrez, P.W., Galán, F., Philips, J., Van Brussel, H., Nuttin, M.: Context-based Filtering for Assisted Brain-Actuated Wheelchair Driving. Computational Intelligence and Neuroscience (2007)

20. Buttfield, A., Ferrez, P.W., Millán, J.d.R.: Towards a Robust BCI: Error Recognition and Online Learning. IEEE Trans. Neural Sys. Rehab. Eng. 14, 164-168 (2006)

21. Millán, J.d.R., Buttfield, A., Vidaurre, C., Krauledat, M., Schögl, A., Shenoy, P., Blankertz, B., Rao, R.P.N., Cabeza, R., Pfurtscheller, G., Müller, K.-R.: Adaptation in BrainComputer Interfaces. In: Dornhege, G., Millán, J.d.R., Hinterberger, T., McFarland, D., Müller, K.-R. (eds.) Towards Brain-Computer Interfacing. MIT Press, Cambridge, Massachusetts (2007)

22. Ferrez, P.W., Millán, J.d.R.: You Are Wrong!-Automatic Detection of Interaction Errors from Brain Waves. In: Proc. 19th International Joint Conference on Artificial Intelligence (2005)

23. Ferrez, P.W., Millán, J.d.R.: Error-Related EEG Potentials in Brain-Computer Interfaces. In: Dornhege, G., Millán, J.d.R., Hinterberger, T., McFarland, D., Müller, K.-R. (eds.) Towards Brain-Computer Interfacing. MIT Press, Cambridge, Massachusetts (2007)

24. Perrin, X., Chavarriaga, R., Siegwart, R., ., Millán, J.d.R.: Bayesian Controller for a Novel Semi-Autonomous Navigation Concept. In: European Conference on Mobile Robotics, Freiburg, Germany (2007) 